\title{
Advances of non-classical crystallization towards self-purification of precious metal nanoparticles mixtures
}

Felizitas Kirner, and Elena V. Sturm*

University of Konstanz, Universitätsstraße 10, 78457 Konstanz, Germany

KEYWORDS: nanoparticle self-assembly, non-classical crystallization, Au@Ag, size and shape separation.

ABSTRACT. Crystallization is a common procedure used for the purification of compounds. In this work, we use particle-mediated crystallization (i.e., "non-classical crystallization") to separate mixtures of Au@Ag nanocrystals of different size and shape into “ordered” phases (i.e., crystalline domains) with nanocrystals of similar morphology and size. The self-assembly process of drying "coffee ring" effect and of depletion forces and the resulting superstructures are investigated. A “depletion" based technique efficiently separates nanoparticle mixtures with small differences in size (12-22\%) within isolated colloidal crystals, while the "coffee ring" technique works better for nanoparticles with a bigger difference in size and shape. Besides the development of a successful approach for the self-purification of nanoparticle mixtures, this study additionally deepens our understanding on particle mediated crystallization under various conditions. 
Most applications of nanoparticulate systems profit from narrow distributions of nanoparticles due to a strong correlation between their physical properties and particle's shape and size. Yet, standard nanocrystal (NC) syntheses oftentimes yield particles with relatively broad size and shape distributions. There are several methods which can be used to purify and classify particle dispersions and narrow their polydispersity in terms of size or shape, including field flow fractionation filtration, chromatography, gel electrophoresis and density gradient centrifugation. ${ }^{1 \text {, }}$ ${ }^{2}$ Particle-mediated crystallization (i.e., "non-classical crystallization”) offers a principial different approach to achieve the same task. ${ }^{3,}{ }^{4}$ Nanoparticle self-assembly to ordered structures and classical crystallization by ions and molecules share some similarities, which makes it possible to use some common techniques and principles to control crystallization processes. One of the easiest methods to purify nanoparticles by self-assembly is a drying-mediated approach where a drop of $\mathrm{NC}$ dispersion is placed on top of a substrate and dried. Depending on interactions between NC and dispersion medium, different structures may evole. ${ }^{5}$ It is postulated that the size separation of colloidal particles (size more than $100 \mathrm{~nm}$ ) within the "coffee ring" occurs due to the geometric constraints at the contact line of the evaporating droplet with the selected substrate. For lyophilic and lyophobic substrates smaller and bigger particles are concentrated in the outer rim, respectively. The "strong" Marangoni flow could scientifically change the particle dynamic within the drying "coffee ring". ${ }^{6-8}$ In 2D films, the so-called "Zenon"-packing describes the selforganization of spheres depending on their size and polydispersity due to size-dispersive van der Waals interactions. ${ }^{9,10}$ A size-dependent phase separation takes place where smaller particles are located on the outer side of the resulting structures. Similar to classical recrystallization method (i.e., repeated crystallization and subsequent dissolution), multiple recrystallizations of colloidal crystals (incl. mesocrystals) allow obtaining particulate systems with narrow size and shape 
distributions. A "self-cleaning" effect of NCs was found for the assembly of Pt NCs, assembly and subsequent washing removes misshaped particles. ${ }^{11}$ In our recent work on cubic iron oxide nanocrystals, we showed that repeated recrystallization of mesocrystals could be successfully used to remove the colloidal impurities and overall tremendously narrow the particle size and shape distribution and achieve a $\mathrm{PDI}_{\mathrm{c}}$ up to $1.0001 .^{3}$

All above mentioned methods were mainly used for either big colloidal particles with sizes more than $100 \mathrm{~nm}$ or relatively small ones with sizes well below $20 \mathrm{~nm}$. Precious metal nanoparticles can be easily synthesized in quite good quality also with sizes between 30 and $100 \mathrm{~nm} .{ }^{12,13} \mathrm{In}$ comparison to smaller nanoparticles, they entail e.g., less Brownian motion and higher sedimentation forces, but still exhibit the tendency to assemble into ordered superstructures (e.g. colloidal crystals, mesocrystals). ${ }^{14}$ For precious metals, self-assembly mediated by depletion forces is a reliable method to generate such $3 \mathrm{D}$ superstructures. ${ }^{14,15}$ A gradual increase of the surfactant concentration of a NC dispersion (e.g., by overlayering the dispersion with a concentrated surfactant solution) leads to destabilization and assembly. ${ }^{15-17}$ Advantage over drying mediated techniques is, among others, the slower self-assembly kinetic, leading to a potentially more ordered structure. However, to our knowledge, for such "intermediate"-sized NCs with a polydispersity in size and shape, a systematic investigation of the crystallization and selfpurification behavior is not yet reported. There are a multitude of simulations predicting the packing behavior of hard colloids, and showing that facet-aligned, locally dense packings of particles are most favored. ${ }^{18-20}$ Yet, in these simulations the only intrinsic forces between the particles are steric and the entropic effects of shape can be isolated and treated separately. ${ }^{20}$ However, in real experimental systems, nanoparticle interactions are difficult to treat separately and their interplay is highly complex..$^{21,22}$ 
To fill the gap, in this communication we present a systematic study where Au@Ag NC (size: 30$62 \mathrm{~nm}$, shape: cubes, rods, etc.) and their mixtures are self-assembled and self-purified by means of a drying-mediated "coffee ring" effect and dispersion-based depletion forces. Non-classical crystallization significantly improves size and shape classification (e.g., separation quality) of nanoparticle dispersion mixtures. The selected precious metal nanoparticles are very suited for such studies as their high density enables the synthesis of monodisperse particles with the same morphology over a large size and weight range.

Five different batches of $\mathrm{Au} @ \mathrm{Ag}$ NCs are synthesized with cubes (edge length: 62 nm, 51 nm, 42 $\mathrm{nm}, 34 \mathrm{~nm}$, and $30 \mathrm{~nm}$ ) as dominating species (75\% cubes, 4\% rods, 21\% others; Figure S3) and dispersion droplets are dried on a polished Si wafer (see Supporting Information for details). The resulting "coffee ring" structures (Figure 1a) show, depending on the composition of the particle batch (Figure 1b), different assembly patterns (Figure 1c-g). For all investigated batches, a clear separation depending on the particle shape occurs. Cubes are assembled in ordered superlattices showing a $\mathrm{p} 4 \mathrm{~mm}$ (or slightly distorted towards $\mathrm{c} 2 \mathrm{~mm}$ ) plain symmetry of the basal plane (parallel to substrate), while rods are forming separate domains and assemble preferably side to side. Particles with similar volumes as cubes but different shape are mainly excluded from both "ordered" phases (i.e., crystalline domains). The rod-like particles that are much larger than the corresponding cubes (rod length $2 \mu \mathrm{m}$, cube edge length $60 \mathrm{~nm}$ ) are mainly assembled at the outer rim of the "coffee ring" pattern (Figure 1c). A transition towards inclusion of rod-clusters within a superlattice of cubic particles occurs for smaller rods, ending with extended vein-like structures of rods within the cube array (Figure 1e). First, this seems contradictive, as for a coffee ring the smaller particles are expected at the outer rim due to capillary forces ${ }^{23}$ and geometric constraints at the contact line of relatively hydrophilic substrate with low contact angle. ${ }^{6-8}$ It could, however, 
be explained by the fact that a contact angle of $43-48^{\circ}$ for dispersion droplets (Figure S6b) is still relatively high for such small nanoparticles and no regions with geometric constrains are formed, which could favor separation of small nanoparticles at the outer rim. Furthermore, larger particles with a higher excluded volume deplete faster as they are less stable in solution due to the higher sedimentation and depletion forces. ${ }^{24,} 25$ The more similar the colloidal stability of the nanoparticles in the dispersion mixture, the more likely is the formation of clusters of rods within the ordered array of cubic particles (Figure $1 \mathrm{c}-\mathrm{g}$ ). The side-by-side orientation of the rods and cubes corresponds to a densest packing with a maximum overlapping volume of the excluded region. ${ }^{24}$ Misshaped particles with no defined packing behavior are excluded to the rim of both phases following self-assembly behavior reported previously for magnetite and Pt NCs. ${ }^{3,4,11} \mathrm{~A}$ more thorough exclusion of misshaped particles occurs for batches with smaller particles size, indicating that a successful particle separation benefits from a higher mobility in solution and a partially reversible attachment to the growing crystalline domains. This is highly influenced by the particle size and strengthens the argument of a partially entropic pathway. 


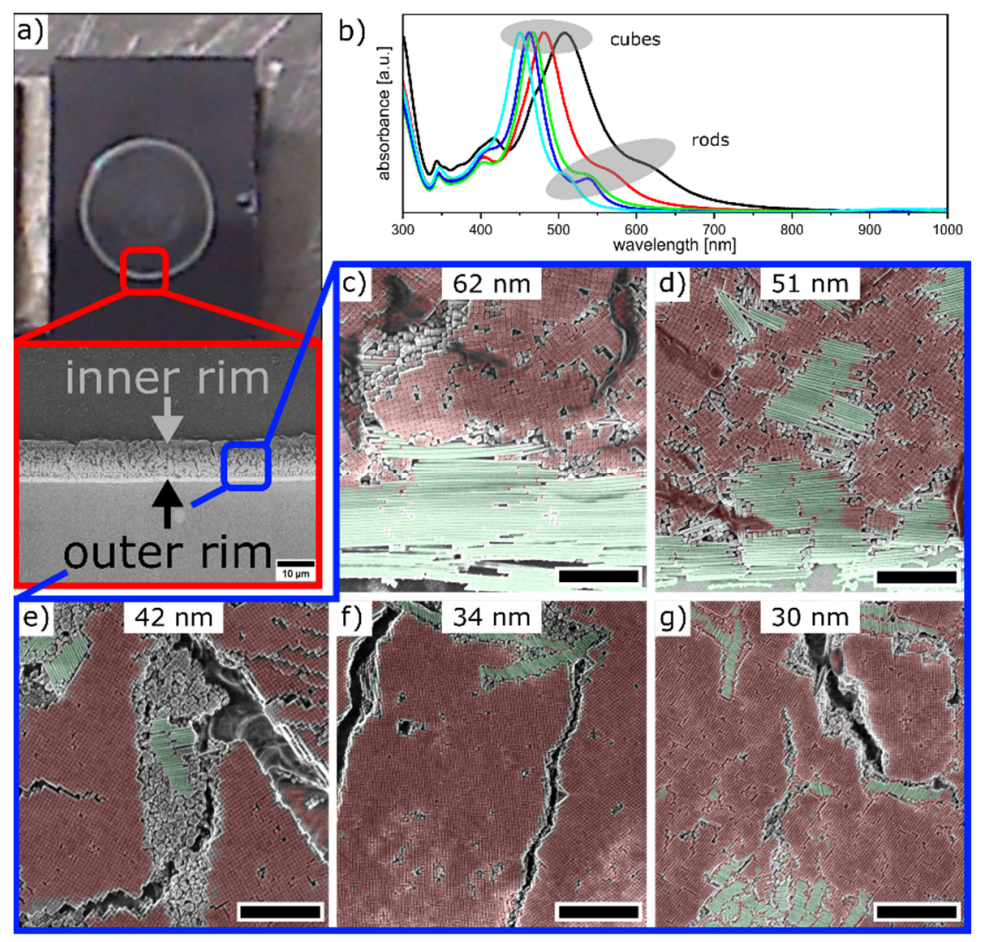

Figure 1.Au@Ag NCs of five different batches are dried on a Si wafer resulting in "coffee ring” structures. a) Photograph and enlarged SEM image of selected region of such a "coffee ring". b) Normalized UV-Vis spectra of the as-prepared NCs. The edge length of the corresponding cubes is $62 \mathrm{~nm}$ (black), $51 \mathrm{~nm}$ (red), $42 \mathrm{~nm}$ (green), $34 \mathrm{~nm}$ (blue), and $30 \mathrm{~nm}$ (cyan). c)-g) SEM images of "coffee rings" of the dried dispersions. The outer rim of the coffee ring is located towards the bottom of the images. Large areas build from cubic particles and rods are highlighted in red or green, respectively. Scale bar is $1 \mu \mathrm{m}$ each. The edge length of the dominant cubic particles is c) $62 \mathrm{~nm}$, d) $51 \mathrm{~nm}$, e) $42 \mathrm{~nm}, \mathrm{f}) 34 \mathrm{~nm}$, and g) $30 \mathrm{~nm}$.

The question that now should be answered is whether there is a separation limit for the proposed approach for mixture of particles with selected differences in sizes or shapes. Therefore, we prepared binary mixtures of nanoparticle batches and repeated the droplet drying process to receive "coffee ring" structures (Figure 2 and Figure 3). The assembly behavior of rods agrees with the one in non-mixed samples with large rods on the outside and smaller ones incorporated (Figure 
S8). A Fourier filtering procedure was applied to each SEM image of a "coffee ring" pattern in order to highlight the crystalline domains of nanoparticles with the same lattice parameters (i.e., assembly from nanoparticles with specific size and shape, Figure 2 and Figure 3). Bandpass masks were applied to the generated fast Fourier transform (FFT) of images at the position of 100 or 200 diffraction rings of the selected superlattices (Figure S9 and Figure S10). Interestingly, no phase separation occurs for particles with small size differences (12-22\%) (cubes of $62 \mathrm{~nm}$ and $51 \mathrm{~nm}$ or $34 \mathrm{~nm}$ and $30 \mathrm{~nm})$, all studied ratios $(2: 1,1: 1$, and 1:2) lead to a single but structurally distorted phase (Figure $2 \mathrm{a} \mathrm{x} \mathrm{b}$ and Figure $3 \mathrm{~d} \times \mathrm{e}$ ). It was found that the lattice parameters of the square lattice of the surface basal plane (Table S2) of the mixed assembly lie in between the one of larger and smaller species while being closer to the one of the higher concentrated particle species. For all other investigated mixtures, the cubes separate in two crystalline phases (Figure 2 and Figure 3) with lattice parameters corresponding to the single species sample independent from the mixing ratio. Here, the bigger nanocubes form crystalline domains assemble close to the outer rim of the "coffee ring", while the smaller ones assembled at the inner rim. We thereby show that a particle mediated crystallization can handle quite a high degree of polydispersity when arranging a crystal lattice. Yet, the sensitivity of the self-assembly process to size-dependent interactions is emphasized as there is no mixed phase observed for combination of smallest and largest particles (e.g., 62 and $30 \mathrm{~nm}$ cubes) where, size-wise, eight small cubes could fill for a large one. This hints towards a critical influence of size-dispersive forces such as Van der Waals interactions. 


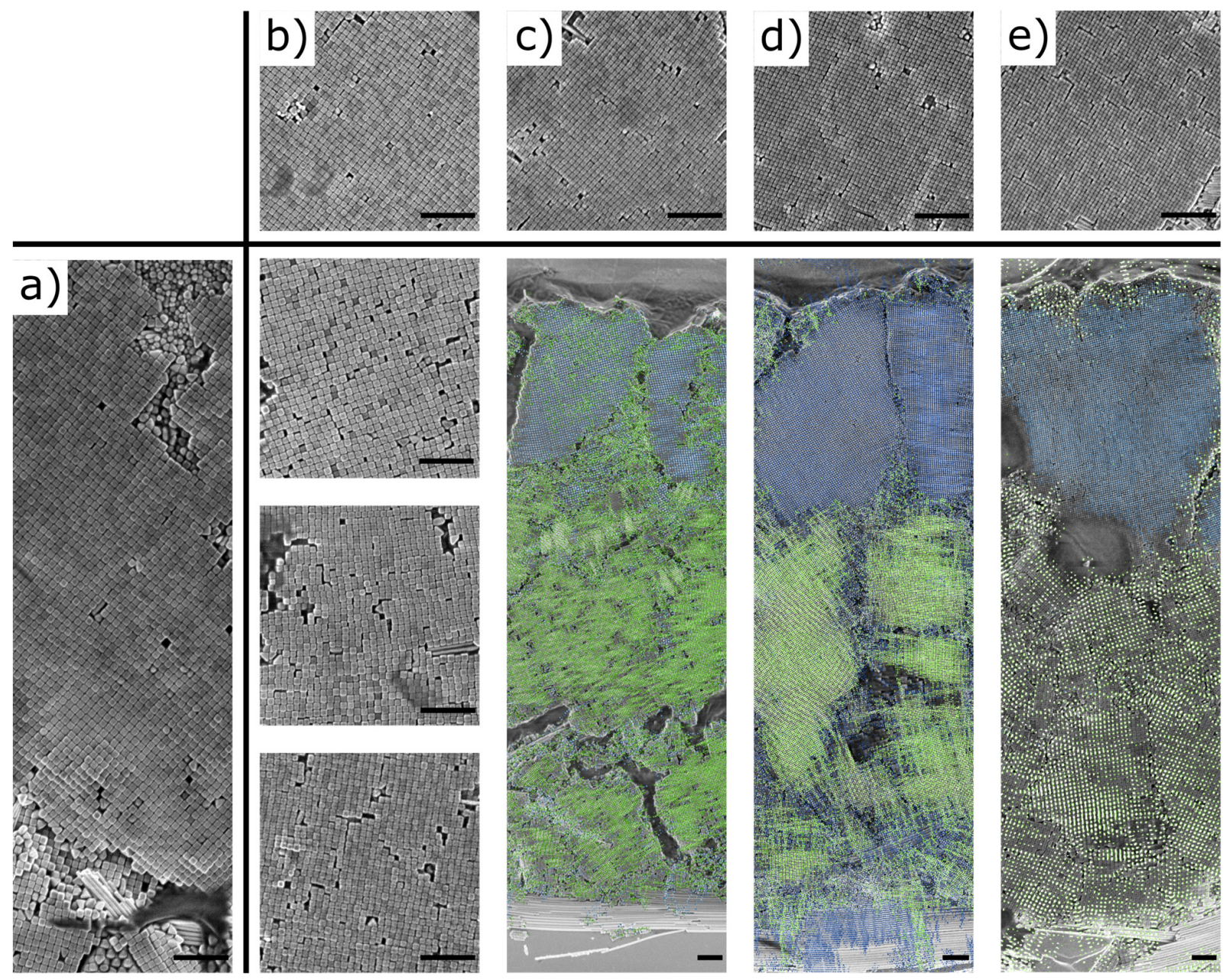

Figure 2. Binary mixtures Au@Ag NCs of five different batches are dried on a Si wafer resulting in "coffee ring” patterns. a-e) Show SEM images of pure particles with NCs of a) $62 \mathrm{~nm}$, b) $51 \mathrm{~nm}$, c) $42 \mathrm{~nm}$, d) 34 $\mathrm{nm}$, and e) $30 \mathrm{~nm}$, respectively. Mixtures of the (a) particles with the respective other sizes are depicted in the according intersection. Mixtures of a) and b) are depicted in the ratios 2:1, 1:1, and 1:2 from top to bottom. The Fourier filtered SEM images show the crystalline domains composed of bigger (green area) and smaller (blue area) nanocubes (mixture ratio: 1:1). Scale bar is $500 \mathrm{~nm}$. 

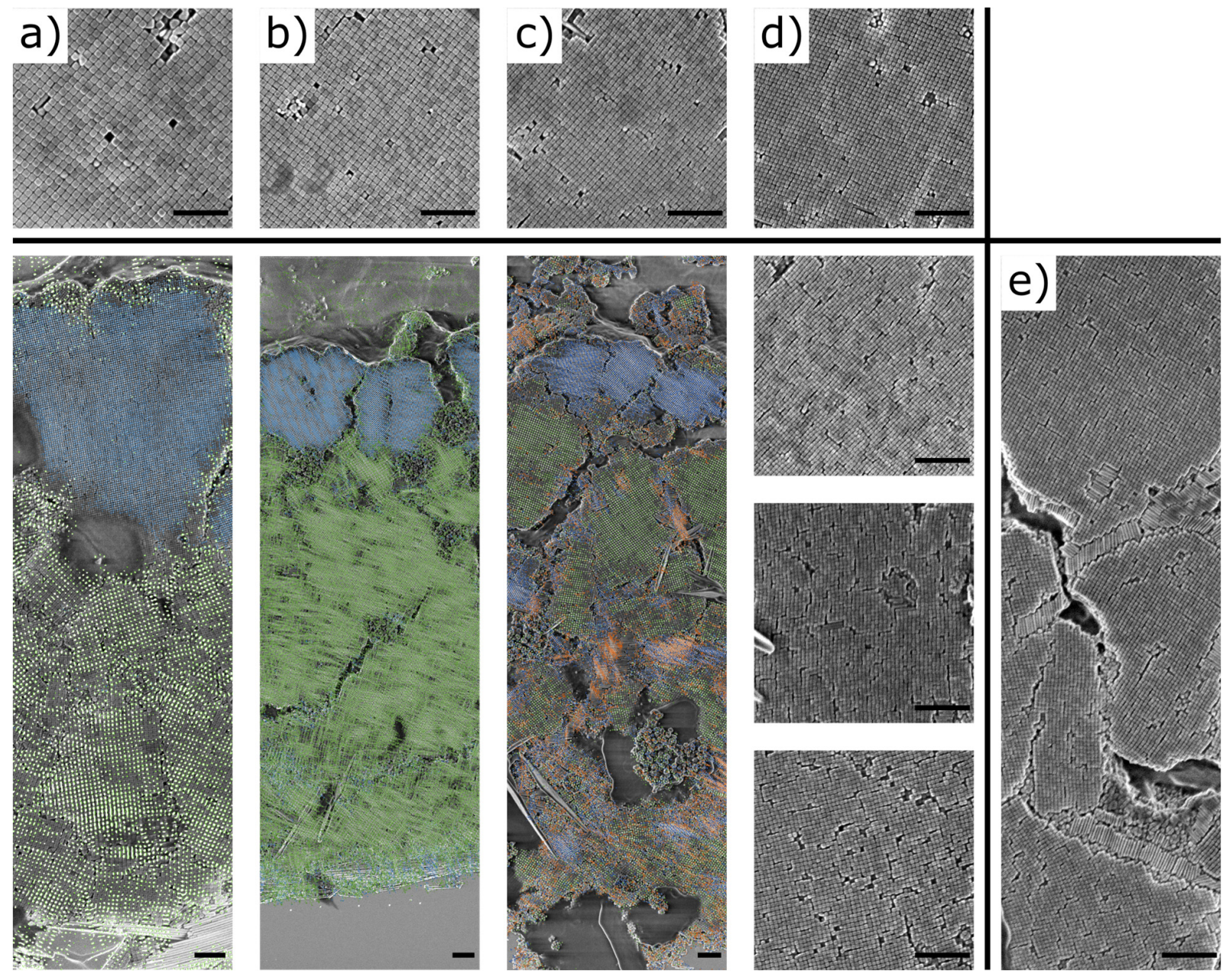

Figure 3. Binary mixtures $\mathrm{Au} @ \mathrm{Ag}$ NCs of five different batches are dried on a Si wafer resulting in "coffee ring" patterns. a-e) Show SEM images of pure particles with NCs of a) $62 \mathrm{~nm}$, b) $51 \mathrm{~nm}$, c) $42 \mathrm{~nm}$, d) 34 $\mathrm{nm}$, and e) $30 \mathrm{~nm}$, respectively. Mixtures of the (e) particles with the respective other sizes are depicted in the according intersection. Mixtures of e) and d) are depicted in the ratios 2;1, 1:1, and 1:2 from top to bottom. The Fourier filtered SEM images show the crystalline domains composed of bigger (green area) and smaller (blue area) nanocubes, and clusters of rod-like particles (orange area) (mixture ratio: 1:1). Scale bar is $500 \mathrm{~nm}$.

To eliminate the effect of evaporation dynamics on the self-assembly process and to further study the influence of the depletion interaction in nanoparticle dispersions, the crystallization process 
was induced by overlayering the stable dispersion with concentrated solution of CTAC (cetylpyridinium chloride) surfactant (Supplementary Movie). This was reported to be an excellent method to generate ordered nanoparticle superstrucures. ${ }^{14,15}$ The nanoparticle self-assembly is induced by diffusion of depletant into the nanoparticle dispersion, and depending on its colloidal stability the complete destabilization occurs within several hours to weeks ( $30 \mathrm{~h}$ for $62 \mathrm{~nm}$ cubes, 14 days for $30 \mathrm{~nm}$ cubes, Experimental Section SI, Figure 4, Supplementary Movie). Figure 4 shows SEM images of resulting nanoparticle assemblies on the silicon substrate. For the largest $(62 \mathrm{~nm})$ cubes no 3D ordered structures can be observed. This may be due to the large mass of nanoparticles and instability against gravitational sedimentation, so that precipitation of nanoparticles was too fast to form ordered assemblies. For all other particle sizes, a major separation into ordered superstructures (also in the form of faceted crystals) built from similarly shaped and sized particles occurs. The surface smoothness of basal faces increases with decreasing particle size emphasizing a higher driving force and growth rate for larger particles. Indeed, larger particles are observed to deplete much faster under otherwise same ambient conditions. Smaller particles additionally generate larger superstructures following the idea that a higher stability (through Brownian motion) and slower assembly process lead to higher ordered structures. The lattice parameters of the built superstructures again relate to the particle size (Table S3) but are significantly smaller than for the dried samples (Table S2). This is also found in literature and may be explained perfectly well with the influence of surfactant on the particle's surface. ${ }^{26}$ For a drying approach, solvent swollen, expanded surfactant chains get closer due to decrease of available space (drying) and lock due to e.g., Van der Waals interactions at small distances. In the entropic depletion approach, the solvent quality is slowly decreased and surfactant coronas contract to reduce contact with poor solvent and then "stick" at smaller distances. 

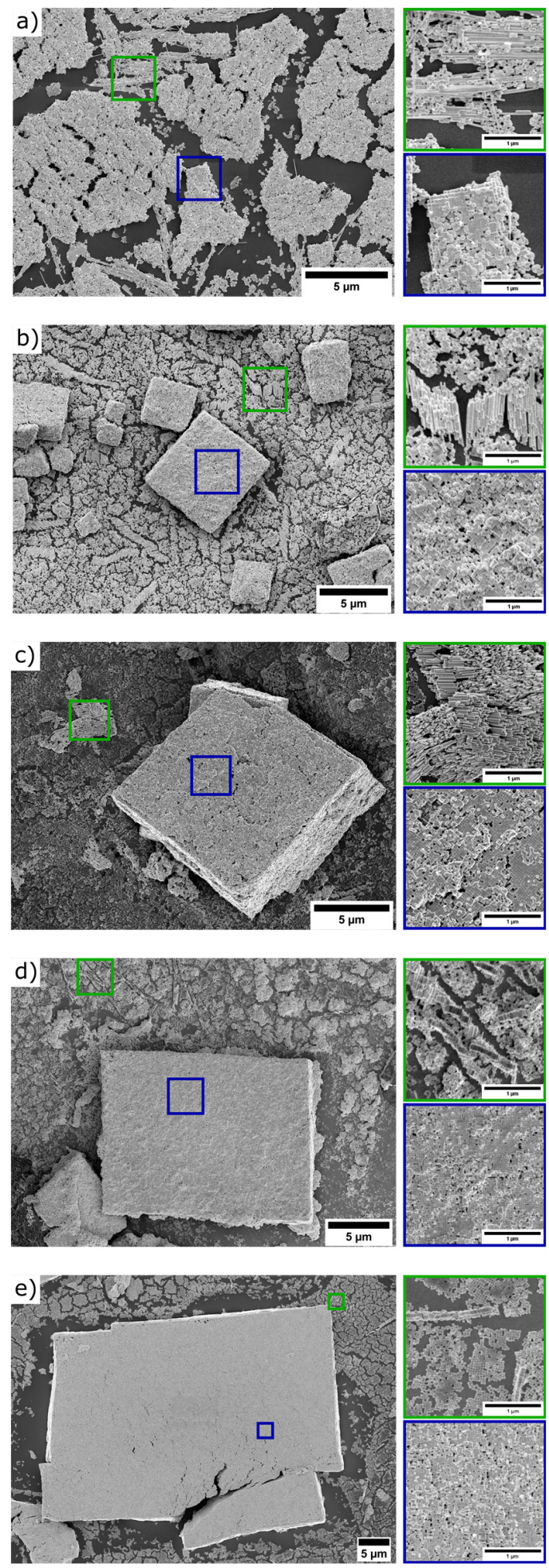

Figure 4. SEM images of depleted self-assemblies of $\mathrm{Au} @$ Ag particles containing cubes of a) $62 \mathrm{~nm}, \mathrm{~b}$ ) $51 \mathrm{~nm}, \mathrm{c}) 42 \mathrm{~nm}$, d) $34 \mathrm{~nm}$, and e) $30 \mathrm{~nm}$ edge length. For b)- e) a major separation of differently shaped 
particles and crystallization into substructures consisting of only cubes occurs, rods etc. are excluded. The blue area enlarges the structures build mainly from cubes and the green area show structures consisting of rods and nanoparticles of arbitrary size/shape. Lattice parameters of the square array can be found in Table S3.

An investigation of the self-assembly of cubes from batch mixtures under depletion condition shows quite surprising results. The batch of the smallest cubes was mixed with ones with other sizes and depleted in the same fashion (Figure 5). It was shown that, in principle, the synthesis conditions allow a generation of ordered superstructures from studied nanoparticles dispersions through depletion as by the drying mediated approach. Initially, it was expected that for the depletion-mediated assembly, the particles with specific size/shape assemble into separate crystalline structures, similar to the drying mediated approach (Figure 2 and Figure 3). However, this does not happen and while there is still a separation of the particle species, 3D faceted colloidal crystals (i.e., mesocrystals) are not built from cubes larger than $34 \mathrm{~nm}$ (Figure $5 \mathrm{a}-\mathrm{c}$ ). The according assemblies are forming monolayers of the larger particle species and faceted colloidal crystals are built of the smallest cubes. This indicates that the smaller particle species plays a major role in the depletion and probably acts (besides the surfactant) as a secondary depletant that destabilized the other species. ${ }^{27}$ Yet, for small size differences (12-22\%) this effect does not take place. In contrast to the drying method where a mixed particle phase occurs, here we gain separate colloidal crystals that are built from one type of particles each (Figure 5d). 

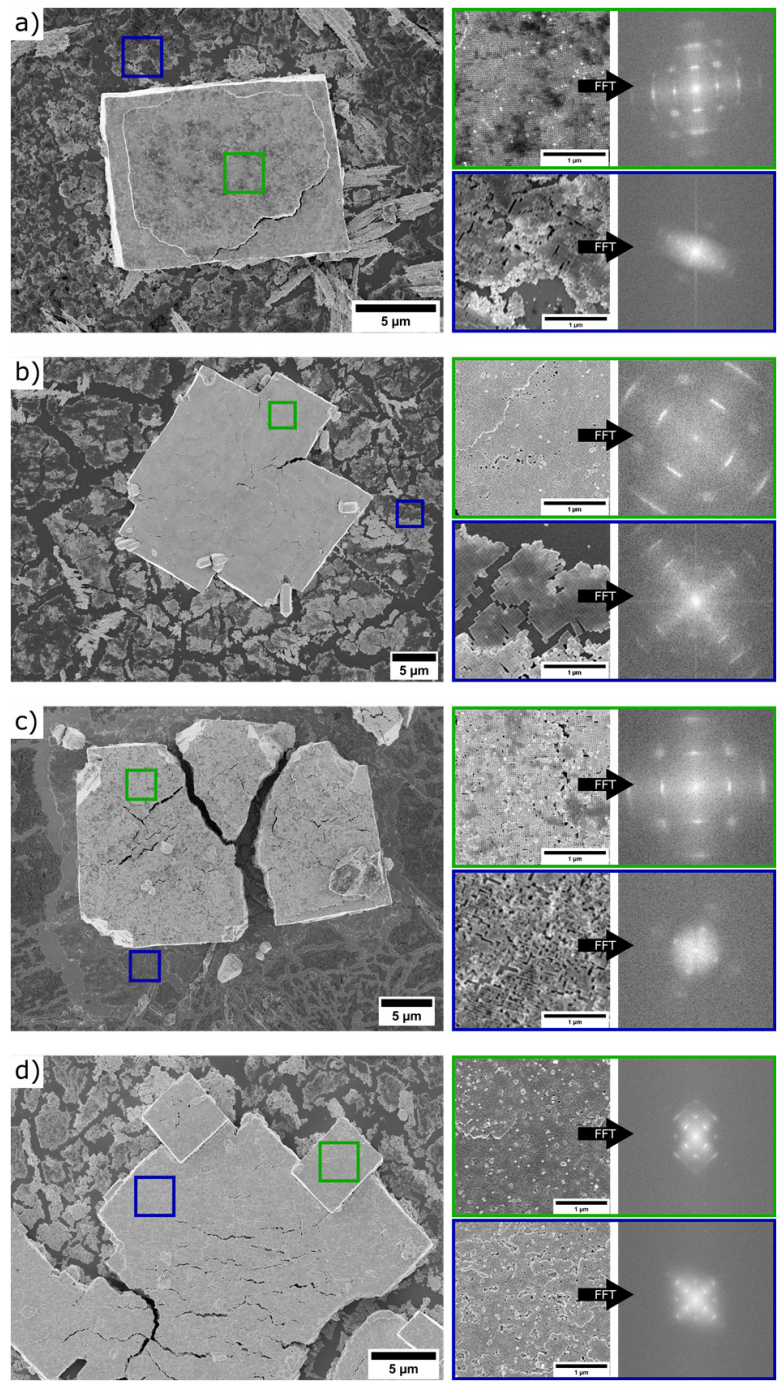

Figure 5. SEM images of depleted self-assemblies of particle mixtures of nanocubes with edge lengths of $30 \mathrm{~nm}$ with a) $62 \mathrm{~nm}$, b) $51 \mathrm{~nm}$, c) $42 \mathrm{~nm}$ and d) $34 \mathrm{~nm}$. Elaborate 3D structures are only generated for species smaller than $34 \mathrm{~nm}$ while the other cubes are mainly assembled in monolayers at the surface of the Si wafer. The green area enlarges the structures build from $30 \mathrm{~nm}$ cubes with respective FFT and the blue area the structures from the respective larger species. Lattice parameters of the square array at the basal plane of the colloidal crystals can be found in Table S3. 
Mixtures with the largest particle species $(62 \mathrm{~nm})$ behave exactly as now predicted: 3D colloidal crystals are built from the respective smaller species with appropriate lattice parameters (Table S3, Figure S11). Similar to the mixture of the two smallest species, phase separation also occurs for the two largest species (Figure S11a). However, in contrast to the previous example, the largest species are mostly destroyed in the assemblies. The largest sample is already quite instable without additional mixing. In mixtures of $62 \mathrm{~nm}$ and $30 \mathrm{~nm}$ cubes the larger species are not destroyed but nicely assembled in monolayers. It may be that the huge difference in assembly kinetics leads to an avoidance of destructive interactions of differently sized particles.

Interestingly the surface roughness and topology are significantly different for colloidal crystals assembled from different nanoparticles under the same crystallization conditions. For classical crystallization, a high driving force results in rough surfaces with an adhesive type growth mechanism $^{28}$ and this type of surface can be observed for larger particles $(51 \mathrm{~nm}, 42 \mathrm{~nm}, 34 \mathrm{~nm}$; Figure S11a-c). Theory predicts a transition to a two-dimensional nucleation growth and further to a spiral growth mechanism for smaller driving forces. This exact spiral growth can now be observed for structures built from the smallest $(30 \mathrm{~nm})$ cubes (Figure 5a-c, Figure S11d). This shows again how fundamental principles of crystal growth can be transferred from classical to non-classical crystallization.

In conclusion, we show a successful self-purification behavior of nanoparticle mixtures according to shape and size of particles by means of the non-classical crystallization. This approach was tested for nanoparticle assemblies induced by a drying-mediated "coffee ring" effect and dispersion-based depletion forces. We found, that for nanoparticle mixtures with small differences in size (12-22\%) the "depletion" based technique allows to efficiently separate the species within isolated colloidal crystals, while the "separation" crystallization in "coffee ring" technique works 
better for nanoparticles with bigger difference in size and shape. This study may provide a basis for further works on the targeted large-scale self-purification of nanoparticles mixtures and further deepens our understanding on particle mediated crystallization and formation of colloidal crystals and mesocrystals.

\section{ASSOCIATED CONTENT}

\section{Supporting Information.}

The following files are available free of charge. Experimental methods: Chemicals and materials, synthesis of Au seeds, synthesis of Au spheres, synthesis of $\mathrm{Au} @ \mathrm{Ag}$ cubes, preparation of $\mathrm{Au} @ \mathrm{Ag}$ self-assembled superstructures and the corresponding characterization (PDF).

Movie S1: Time lapse of a depletion-mediated $\mathrm{Au@Ag} \mathrm{self-assembly} \mathrm{process} \mathrm{at} \mathrm{the} \mathrm{example} \mathrm{of}$ the $62 \mathrm{~nm}$ cube sample (mp4).

\section{AUTHOR INFORMATION}

\section{Corresponding Author}

*E-Mail: Elena V. Sturm: elena.sturm@uni-konstanz.de

\section{Author Contributions}

The manuscript was written through contributions of all authors. All authors have given approval to the final version of the manuscript. 


\section{Funding Sources}

DFG (Deutsche Forschungsgemeinschaft) SFB 1214, Project B1; Zukunfskolleg at the University of Konstanz.

\section{Notes}

The authors declare no competing financial interest.

ACKNOWLEDGMENT We hereby acknowledge the DFG (Deutsche Forschungsgemeinschaft) for financial support (SFB 1214. Project B1). E.S. thanks the Zukunftskolleg at the University of Konstanz and "Konstanzia Transition" programme of equal opportunity council for financial support.

SYNOPSIS

\section{non-classical crystallization}

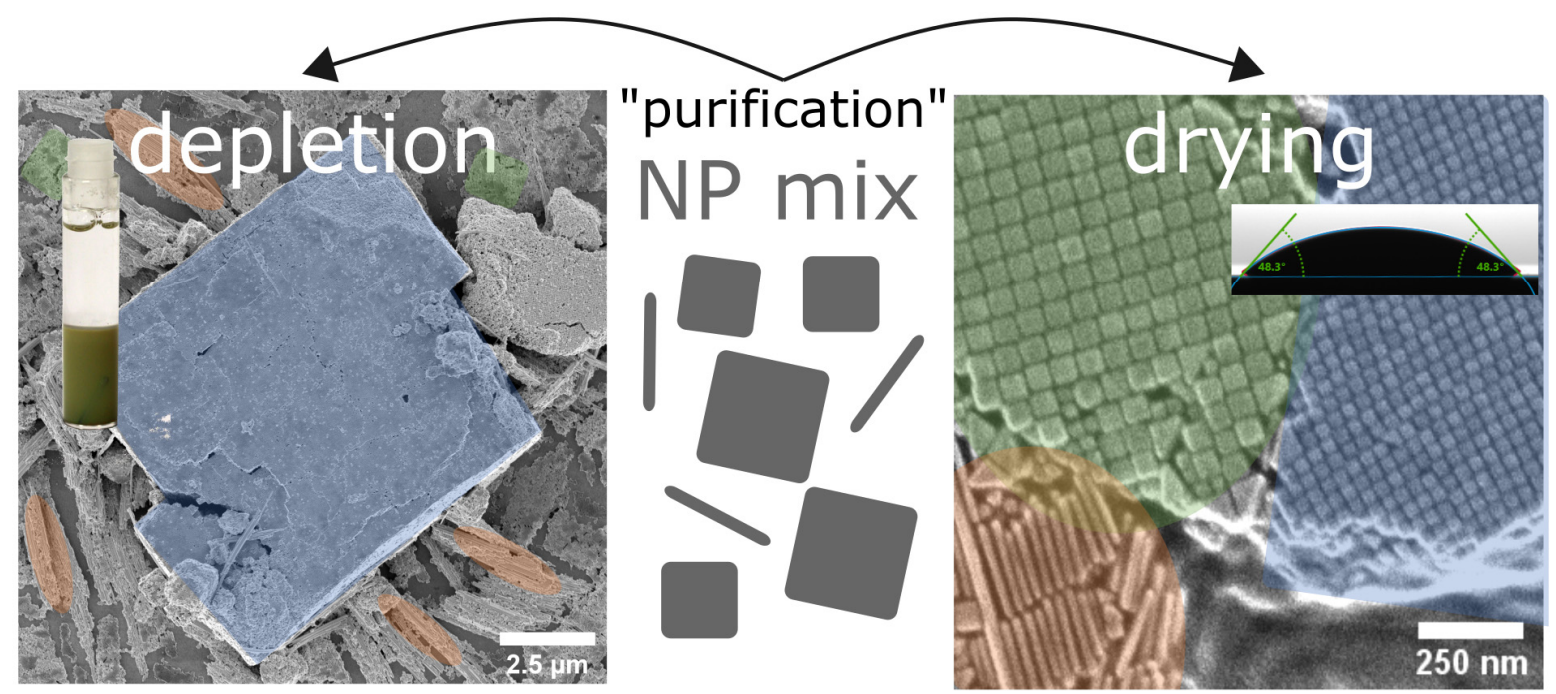




\section{REFERENCES}

(1) Robertson, J. D.; Rizzello, L.; Avila-Olias, M.; Gaitzsch, J.; Contini, C.; Magoń, M. S.; Renshaw, S. A.; Battaglia, G., Purification of Nanoparticles by Size and Shape. Scientific Reports 2016, 6, (1), 27494.

(2) Süß, S.; Bartsch, K.; Wasmus, C.; Damm, C.; Segets, D.; Peukert, W., Chromatographic property classification of narrowly distributed ZnS quantum dots. Nanoscale 2020, 12, (22), 12114-12125.

(3) Brunner, J. J.; Maier, B.; Rosenberg, R.; Sturm, S.; Cölfen, H.; Sturm, E., Non-classical Recrystallization. Chemistry - A European Journal 2020, n/a, (n/a).

(4) Josten, E.; Angst, M.; Glavic, A.; Zakalek, P.; Rücker, U.; Seeck, O. H.; Kovács, A.; Wetterskog, E.; Kentzinger, E.; Dunin-Borkowski, R. E.; Bergström, L.; Brückel, T., Strong size selectivity in the self-assembly of rounded nanocubes into 3D mesocrystals. Nanoscale Horizons 2020, 5, (7), 1065-1072.

(5) Kletenik-Edelman, O.; Ploshnik, E.; Salant, A.; Shenhar, R.; Banin, U.; Rabani, E., Drying-Mediated Hierarchical Self-Assembly of Nanoparticles: A Dynamical Coarse-Grained Approach. The Journal of Physical Chemistry C 2008, 112, (12), 4498-4506.

(6) Yi, J.; Jeong, H.; Park, J., Modulation of nanoparticle separation by initial contact angle in coffee ring effect. Micro and Nano Systems Letters 2018, 6, (1), 17.

(7) Jeong, H.; Tiem, J.; Gianchandani, Y.; Park, J., Nano-particle separation using Marangoni flow in evaporating droplets. ed.; 2014.

(8) Wong, T.-S.; Chen, T.-H.; Shen, X.; Ho, C.-M., Nanochromatography Driven by the Coffee Ring Effect. Analytical Chemistry 2011, 83, (6), 1871-1873.

(9) Ohara, P. C.; Leff, D. V.; Heath, J. R.; Gelbart, W. M., Crystallization of opals from polydisperse nanoparticles. Phys Rev Lett 1995, 75, (19), 3466-3469.

(10) Antonietti, M.; Hartmann, J.; Neese, M.; Seifert, U., Highly Ordered Size-Dispersive Packings of Polydisperse Microgel Spheres. Langmuir 2000, 16, (20), 7634-7639.

(11) Kang, Y.; Li, M.; Cai, Y.; Cargnello, M.; Diaz, R. E.; Gordon, T. R.; Wieder, N. L.; Adzic, R. R.; Gorte, R. J.; Stach, E. A.; Murray, C. B., Heterogeneous Catalysts Need Not Be so "Heterogeneous": Monodisperse Pt Nanocrystals by Combining Shape-Controlled Synthesis and Purification by Colloidal Recrystallization. Journal of the American Chemical Society 2013, 135, (7), 2741-2747.

(12) Kirner, F.; Potapov, P.; Schultz, J.; Geppert, J.; Müller, M.; González-Rubio, G.; Sturm, S.; Lubk, A.; Sturm, E., Additive-controlled synthesis of monodisperse single crystalline gold nanoparticles: interplay of shape and surface plasmon resonance. Journal of Materials Chemistry C 2020, 8, (31), 10844-10851.

(13) Gómez-Graña, S.; Goris, B.; Altantzis, T.; Fernández-López, C.; Carbó-Argibay, E.; Guerrero-Martínez, A.; Almora-Barrios, N.; López, N.; Pastoriza-Santos, I.; Pérez-Juste, J.; Bals, S.; Van Tendeloo, G.; Liz-Marzán, L. M., Au@Ag Nanoparticles: Halides Stabilize $\{100\}$ Facets. The Journal of Physical Chemistry Letters 2013, 4, (13), 2209-2216.

(14) Henzie, J.; Grunwald, M.; Widmer-Cooper, A.; Geissler, P. L.; Yang, P., Self-assembly of uniform polyhedral silver nanocrystals into densest packings and exotic superlattices. Nat Mater 2011, 11, (2), 131-7.

(15) Yang, C.-W.; Chiu, C.-Y.; Huang, M. H., Formation of Free-Standing Supercrystals from the Assembly of Polyhedral Gold Nanocrystals by Surfactant Diffusion in the Solution.

Chemistry of Materials 2014, 26, (16), 4882-4888. 
(16) Yang, P. W.; Thoka, S.; Lin, P. C.; Su, C. J.; Sheu, H. S.; Huang, M. H.; Jeng, U. S., Tracing the Surfactant-Mediated Nucleation, Growth, and Superpacking of Gold Supercrystals Using Time and Spatially Resolved X-ray Scattering. Langmuir 2017, 33, (13), 3253-3261.

(17) Huang, M. H.; Thoka, S., Formation of supercrystals through self-assembly of polyhedral nanocrystals. Nano Today 2015, 10, (1), 81-92.

(18) Damasceno, P. F.; Engel, M.; Glotzer, S. C., Predictive Self-Assembly of Polyhedra into Complex Structures. Science 2012, 337, (6093), 453-457.

(19) van Anders, G.; Ahmed, N. K.; Smith, R.; Engel, M.; Glotzer, S. C., Entropically patchy particles: engineering valence through shape entropy. ACS Nano 2014, 8, (1), 931-40.

(20) van Anders, G.; Klotsa, D.; Ahmed, N. K.; Engel, M.; Glotzer, S. C., Understanding shape entropy through local dense packing. Proc Natl Acad Sci U S A 2014, 111, (45), E4812-21. (21) Bishop, K. J. M.; Wilmer, C. E.; Soh, S.; Grzybowski, B. A., Nanoscale Forces and Their Uses in Self-Assembly. Small 2009, 5, (14), 1600-1630.

(22) Min, Y.; Akbulut, M.; Kristiansen, K.; Golan, Y.; Israelachvili, J., The role of interparticle and external forces in nanoparticle assembly. Nat Mater 2008, 7, (7), 527-38.

(23) Mampallil, D.; Eral, H. B., A review on suppression and utilization of the coffee-ring effect. Adv Colloid Interface Sci 2018, 252, 38-54.

(24) Petukhov, A. V.; Tuinier, R.; Vroege, G. J., Entropic patchiness: Effects of colloid shape and depletion. Current Opinion in Colloid \& Interface Science 2017, 30, 54-61.

(25) Lekkerkerker, H. N. W.; Tuinier, R., Lecture Notes in Physics. In Colloids and the depletion interaction, Springer: 2011; Vol. 833, pp 57-108.

(26) Lee, B.; Podsiadlo, P.; Rupich, S.; Talapin, D. V.; Rajh, T.; Shevchenko, E. V.,

Comparison of Structural Behavior of Nanocrystals in Randomly Packed Films and Long-Range Ordered Superlattices by Time-Resolved Small Angle X-ray Scattering. Journal of the American Chemical Society 2009, 131, (45), 16386-+.

(27) Rossi, L.; Soni, V.; Ashton, D. J.; Pine, D. J.; Philipse, A. P.; Chaikin, P. M.; Dijkstra, M.; Sacanna, S.; Irvine, W. T. M., Shape-sensitive crystallization in colloidal superball fluids. Proceedings of the National Academy of Sciences 2015, 112, (17), 5286-5290.

(28) Sunagawa, I., Crystals. ed.; Cambridge University Press: Cambridge, 2009. 